\title{
Algorithms for graphs embedable with few crossings per egde
}

Citation for published version (APA):

Grigoriev, A., \& Bodlaender, H. L. (2004). Algorithms for graphs embedable with few crossings per egde. METEOR, Maastricht University School of Business and Economics. METEOR Research Memorandum No. 035 https://doi.org/10.26481/umamet.2004035

Document status and date:

Published: 01/01/2004

DOI:

10.26481/umamet.2004035

Document Version:

Publisher's PDF, also known as Version of record

\section{Please check the document version of this publication:}

- A submitted manuscript is the version of the article upon submission and before peer-review. There can be important differences between the submitted version and the official published version of record.

People interested in the research are advised to contact the author for the final version of the publication, or visit the DOI to the publisher's website.

- The final author version and the galley proof are versions of the publication after peer review.

- The final published version features the final layout of the paper including the volume, issue and page numbers.

Link to publication

\footnotetext{
General rights rights.

- You may freely distribute the URL identifying the publication in the public portal. please follow below link for the End User Agreement:

www.umlib.nl/taverne-license

Take down policy

If you believe that this document breaches copyright please contact us at:

repository@maastrichtuniversity.nl

providing details and we will investigate your claim.
}

Copyright and moral rights for the publications made accessible in the public portal are retained by the authors and/or other copyright owners and it is a condition of accessing publications that users recognise and abide by the legal requirements associated with these

- Users may download and print one copy of any publication from the public portal for the purpose of private study or research.

- You may not further distribute the material or use it for any profit-making activity or commercial gain

If the publication is distributed under the terms of Article $25 \mathrm{fa}$ of the Dutch Copyright Act, indicated by the "Taverne" license above, 


\title{
Algorithms for graphs embeddable with few crossings per edge*
}

\author{
Hans L. Bodlaender ${ }^{\dagger} \quad$ Alexander Grigoriev ${ }^{\ddagger}$
}

\begin{abstract}
We consider graphs that can be embedded on a surface of bounded genus such that each edge has a bounded number of crossings. We prove that many optimization problems, including maximum independent set, minimum vertex cover, minimum dominating set and many others, admit polynomial time approximation schemes when restricted to such graphs. This extends previous results by Baker [1] and Eppstein [3] to a much broader class of graphs.
\end{abstract}

\section{Introduction}

Already more than two decades ago, Baker [1] showed that the maximum independent set and many other NP-hard optimization problems on graphs admit polynomial time approximation schemes (PTAS) when restricted to planar graphs. The basic idea of Baker's algorithm was to remove the vertices in every $k$ th level of a breadth first search tree (BFS) and to solve the problem on the remaining components by a dynamic programming algorithm. Baker proved that from $k$ ways of choosing which set of levels to remove there is at least one which only decreases the size of the maximum independent set by a factor of at most $(k-1) / k$. Moreover, remaining components after levels deletion are $k$-outerplanar graphs, and dynamic programming can solve the problem on these components efficiently.

Recently, Eppstein in [3] observed that the results by Baker [1] can be extended to any minor-closed family of graphs satisfying so-called diametertreewidth property. This implies that the problem admits a PTAS if restricted to bounded-genus graphs. This result has been generalized to other minorclosed classes; in particular, Grohe gave PTAS's for several problems, for any minor-closed family that does not contain all graphs [2].

In this paper we continue the line of investigations - in which way can Baker's technique be further extended? Revisiting Eppstein [3] result, we observe that the restriction that the class of graphs must be minor-closed can be relaxed. By moving from the input graph to an auxiliary graph obtained by

\footnotetext{
*This research was supported by the Netherlands Organization for Scientific Research NWO.

${ }^{\dagger}$ Institute of Information and Computing Sciences, Utrecht University, Padualaan 14, De Uithof, P.O. Box 80089, 3508 TB Utrecht, The Netherlands, Email: hansb@cs.uu.nl

${ }^{\ddagger}$ Maastricht University, Faculty of Economics and Business Administration, Quantitative Economics, P.O. Box 616, 6200 MD Maastricht, The Netherlands, Email: a.grigoriev@ke.unimaas.nl
} 
replacing each crossing by a vertex and back, we can obtain Baker-type PTAS's for several problems on graph that are embeddable on a surface of bounded genus (e.g., the plane) with a bounded number of crossings per edge.

\section{Problem and Definitions}

We illustrate the basic ideas of the PTAS on the maximum independent set problem. Given a graph $G=(V, E)$, we look for a maximum cardinality independent set in $G$, i.e. a vertex subset $V^{\prime} \subseteq V$ such that no two vertices from $V^{\prime}$ are adjacent by an edge from $E$. Let $n=|V|$. This problem is known to be NP-hard even for planar graphs. The problem admits a PTAS if restricted to planar graphs [1] and even to bounded-genus graphs [3].

Definition 2.1 (Good embedding). We call an embedding of graph $G$ on a surface $S$ of genus $g$ a good embedding if it satisfies the following conditions: (i) all vertices of the graph are given as distinct points in $S$; (ii) no two edge crossings happen in the same point in $S$; (iii) for any edge no vertex of the graph, except the endpoints of the edge, is situated on the edge.

Definition 2.2 (Crossing parameter). Let the crossing parameter $\varphi$ of a graph (on surface $S$ ) be the minimum over all good embeddings on $S$ of the maximum over all edges e of the number of edge crossings of $e$.

Through this paper we assume that a good embedding of $G$ is given and both the crossing parameter $\varphi$ and the genus $g$ of $S$ are bounded by some constants. Clearly, the graph is planar if $g=0$ and $\varphi=0$.

\section{The Polynomial Time Approximation Scheme}

We now describe our polynomial time approximation scheme for the maximum independent set problem on graphs with bounded crossing parameter on bounded genus. Consider the following algorithm $\mathcal{A}$ which is a revised version of the algorithms by Baker [1] and Eppstein [3].

Construct the graph $G^{\prime}=\left(V^{\prime}, E^{\prime}\right)$ obtained from $G$ by replacing each edge crossing by a vertex. W.l.o.g. let $\varphi<k$. Build an arbitrary breadth first search tree $T$ of $G$, with root $v_{0}$, and consider the levels of the tree (i.e., vertex sets with equal distance to $v_{0}$ ). Suppose we remove every $k$ th level in $T$ from $G^{\prime}$ together with its $\varphi$ successive levels. This decomposes $G^{\prime}$ into a collection of subgraphs $\mathcal{H}=\left\{H_{1}, H_{2}, \ldots, H_{r}\right\}$ where each subgraph $H_{t}=\left(V_{t}, E_{t}\right)$ is induced by $k-\varphi-1$ consecutive levels in $T$ of $G^{\prime}$.

Consider a subgraph $G_{t}$ of $G$ induced by vertices $V_{t} \cap V$. Since number of crossings per edge is at most $\varphi$ and we removed $\varphi+1$ consecutive levels from $G^{\prime}$, we have that after deletion of levels there is no an edge $e \in E$ such that its two endpoints belong to two different subgraphs $G_{t^{\prime}}$ and $G_{t^{\prime \prime}}$. Therefore, for each choice of $k$ we have a subgraph of $G$ formed by a collection of subgraphs $G_{1}, G_{2}, \ldots, G_{r}$. By arguments similar to Baker's [1] there is a choice of the deleting set of levels which leads to a subgraph approximating the optimum within a factor $(k-\varphi-1) / k$. 
Now, let us show that the treewidth of $G_{t}$ is bounded by $O(k)$ for all $t=$ $1, \ldots, r$. Consider a subgraph $H_{t}$ induced by levels $r+1, r+2, \ldots, r+s$ in $T$ of $G^{\prime}$ where $s=k-\varphi-1=O(k)$. Consider a minor of $G^{\prime}$ obtained by contraction of the first $r$ levels in $T$ to a single vertex and deletion of all levels above $r+s$. Clearly, this minor is a graph of genus $g$. Moreover, it has a diameter of at most $2(k-\varphi-1)=O(k)$. By Eppstein [3] the treewidth of such a minor is $O(g k)$. Therefore, $H_{t}$ as a subgraph of such a minor has the treewidth of at most $O(g k)$ as well.

Now, let us estimate how much the treewidth of $G_{t}$ and $H_{t}$ can differ. Construct a graph $H_{t}^{\prime}$ from $H_{t}$ by replacing each vertex $v$ in $H_{t}$ that represents an edge crossing, say $e_{1}$ and $e_{2}$, by two adjacent vertices $v_{1}$ and $v_{2}$ representing $e_{1}$ and $e_{2}$ respectively. Let $v_{1}$ be adjacent to all vertices corresponding to the neighborhood of $v$ representing $e_{1}$, and let $v_{2}$ be adjacent to all vertices corresponding to the neighborhood of $v$ representing $e_{2}$. It is not hard to see that a tree decomposition of $H_{t}$ of treewidth $d$ can be turned into a tree decomposition of $H_{t}^{\prime}$ of treewidth at most $2 d+1$. One can also observe that we can select for each edge in $G_{t}$ a path in $H_{t}^{\prime}$ between its endpoints, such that these paths do not have internal vertices in common. Thus, $G_{t}$ is a minor of $H_{t}^{\prime}$ and hence the treewidth of $G_{t}$ is at most twice the treewidth of $H_{t}$ plus one, and thus $O(g k)=O(k)$ as required. Hence, the maximum independent set for $G_{t}$ can be found in time $O\left(n 2^{O(k)}\right)$ by a dynamic programming algorithm, using standard treewidth techniques. As a result we have the following theorem.

Theorem 3.1. Algorithm $\mathcal{A}$ outputs an independent set of graph $G$ of size at least $1-O(1 / k)$ times the optimum in time $O\left(k n 2^{O(k)}\right)$, and thus, there is a PTAS for maximum independent set for graphs given with an embedding on a surface of bounded genus and with bounded crossing parameter.

For each of the following problems (and many others), we can also obtain in a similar way, using techniques similar to those of Baker [1], a PTAS for graphs embeddable on a surface of bounded genus with bounded crossing parameter: minimum vertex cover, minimum dominating set, minimum edge dominating set, minimum triangle matching, maximum $H$-matching, maximum tile salvage.

\section{More on the Crossing Parameter}

In this section we give some results on the computational complexity of the crossing parameter and some properties of the class of graphs with bounded crossing parameter.

Theorem 4.1. The problem to determine if a given graph $G$ can be embedded on the plane with crossing parameter 1 is NP-complete.

Corollary 4.1.1. When $P \neq N P$, there does not exist a polynomial time 2approximation algorithm for finding the crossing parameter of a graph on the plane.

Notice, however, that several natural classes of graphs have a bounded crossing parameter on the plane. For instance, graphs of intersections of objects in 
the plane with bounded objects density (disk graphs with bounded density are special case of these); graphs with bounded degree and bounded tree width; planar graphs.

Observation 4.1. The class of graphs with an embedding on the plane with crossing parameter 1 is not closed under taking minors. In fact, every graph is a minor of a graph with crossing parameter 1: take any good embedding, and then add a new vertex of degree two between every two successive crossings.

From work on the crossing number of graphs (the minimum total number of crossings in a planar embedding), we can also obtain bounds on the crossing parameter (on the plane). E.g., the crossing number of a complete graph with $n$ vertices is $\Theta\left(n^{4}\right)$ [4], hence its crossing parameter is $\Theta\left(n^{2}\right)$.

\section{References}

[1] B. Baker. Approximation algorithms for NP-complete problems on planar graphs. Journal of the ACM 41 (1994), 153-180. Preliminary version in FOCS'83.

[2] Martin Grohe, Local tree-width, excluded minors, and approximation algorithms. Combinatorica 23 (2003), 613-632.

[3] D. Eppstein. Diameter and treewidth in minor-closed graph families. Algorithmica 27 (2000) 275-291.

[4] F.T. Leighton. New lower bound techniques for VLSI. Mathematical Systems Theory 17 (1984) 47-70. 